\title{
NIVELES DE POTASIO SÉRICOY ESTUDIO ELECTROCARDIOGRÁFICO EN CRÍAS DE ALPACA (Vicugna pacos)
}

\author{
Serum Potassium Levels and Electrcardiographic Study in Young Alpacas \\ (Vicugna pacos)
}

\author{
Roberto Dávila F. ${ }^{1,5}$, Miluska Navarrete Z. ${ }^{2}$, Boris Lira M. ${ }^{3}$, María Vásquez C. ${ }^{3}$, \\ Manuel Paredes P. ${ }^{1}$, José Rodríguez G. ${ }^{4}$
}

\section{Resumen}

El presente estudio tuvo por objetivo determinar los niveles de potasio sérico y los parámetros electrocardiográficos. El trabajo se hizo en 30 alpacas (17 machos y 13 hembras) menores de 60 días de edad, provenientes de las comunidades alpaqueras de Maranganí, Canchis, en Cusco. El nivel de potasio sérico fue de $5.91 \pm 1.48 \mathrm{mmol} / \mathrm{L}$, sin diferencias estadísticas entre sexos. Los valores electrocardiográficos fueron: Duración onda P, $0.04 \pm 0.0 \mathrm{~s}$; amplitud onda P, $0.173 \pm 0.058 \mathrm{mV}$; duración PQ, $0.101 \pm 0.020 \mathrm{~s}$; duración QRS, $0.038 \pm 0.006 \mathrm{~s}$; complejo QRS, $0.557 \pm 0.412 \mathrm{mV}$; duración QT, $0.237 \pm 0.040 \mathrm{~s}$; amplitud onda T, $0.413 \pm 0.266 \mathrm{mV}$. Asimismo, se observó que crías con onda T aumentada presentaban valores de potasio por encima de $5.5 \mathrm{mmol} / \mathrm{L}$. Además, los valores del potasio sérico y de los parámetros electrocardiográficos fueron similares a los valores de otras especies.

Palabras clave: crías de alpaca, potasio sérico,electrocardiografía

\section{Abstract}

The aim of this study was to determine the levels of serum potassium and electrocardiographic parameters in young alpacas. Thirty alpacas (17 males and 13 females) from 1 to 60 days of age from various alpaca farms in Maranganí, Cusco were used. The levels of serum potassium was $5.91 \pm 1.48 \mathrm{mmol} / \mathrm{L}$ and without statistical differences between sexes. The electrocardiographic values were: $\mathrm{P}$ wave time, $0.04 \pm 0.0 \mathrm{~s}$; $\mathrm{P}$ wave

${ }^{1}$ Clínica de Animales Menores, ${ }^{2}$ Laboratorio de Anatomía Animal y Fauna Silvestre, ${ }^{3}$ Laboratorio de Fisiología Animal, Facultad de Medicina Veterinaria, Universidad Nacional Mayor de San Marcos, Lima

${ }^{4}$ Centro de Investigación IVITA, El Mantaro. Universidad Nacional Mayor de San Marcos, Huancayo

${ }^{5}$ E-mail: rodaf45@yahoo.com

Recibido: 7 de enero de 2014

Aceptado para publicación: 2 de mayo de 2014 
amplitude, $0.173 \pm 0.058 \mathrm{mV}$; PQ time, $0.101 \pm 0.02 \mathrm{~s}$; QRS time, $0.038 \pm 0.006 \mathrm{~s}$; QRS complex, $0.557 \pm 0.412 \mathrm{mV}$; QT time, $0.237 \pm 0.040 \mathrm{~s}$; T wave amplitude, $0.413 \pm 0.266 \mathrm{mV}$. Moreover, animals with increased $\mathrm{T}$ wave had potassium values above $5.5 \mathrm{mmol} / \mathrm{L}$ but without signs of heart disease. In addition, values of serum potassium and electrocardiographic paramters showed similarity to the values of other species.

Key words: baby alpaca, serum potassium, electrocardiography

\section{INTRODUCCIÓN}

El potasio es importante para la función cardiaca. Los pequeños cambios en los niveles de potasio pueden tener un gran efecto en la actividad de nervios y músculos, especialmente el corazón. Los niveles bajos de potasio ocasionan un aumento de la actividad del músculo cardíaco, pudiendo ocasionar latidos cardíacos irregulares, en tanto que los niveles altos causan una disminución de dicha actividad (Seifter, 2012).

El electrocardiograma registra la actividad eléctrica cardíaca, recogida en la superficie del cuerpo. Es una herramienta diagnóstica, cuyo registro se obtiene al colocar al paciente en estación y en decúbito lateral derecho, sujetando los miembros, sin que entren en contacto entre sí. Una dificultad a tener en cuenta es que, quien sujeta los miembros del paciente interfiere en el trazado, ya que los miembros actúan como conductores de los fenómenos eléctricos cardíacos (Kittleson y Kienle, 1998; Nelson y Couto, 1995).

Martínez et al. (1988), con el uso de un equipo portátil, hicieron registros electrocardiográficos de derivaciones bipolares y unipolares aumentadas a camélidos sudamericanos. De estos, un grupo de 34 ejemplares (alpacas, llamas, vicuñas y guanacos) estuvo en la región Alto-Andina de la I Región de Chile, y otro grupo de 18 individuos compuesto por las cuatro especies, estaban viviendo un mínimo de dos años a nivel del mar, en la $\mathrm{V}$ Región de Chile. El análisis de los resultados demostró ausencia de modificaciones electrocardiográficas inducidas por la condición de altura, salvo una menor frecuencia cardiaca y una mayor y significativa duración de la sístole eléctrica ventricular (Q-TC) en los altiplánicos. Los resultados sugieren que la expresión electrocardiográfica de la actividad cardíaca en los camélidos sudamericanos podría constituir un patrón fisiológico de elevada fijación genética, no modificable significativamente con los comprobados cambios anatómicos cardíacos por efecto de diferencias de altura sobre el nivel del mar.

El objetivo del presente estudio fue determinar los niveles de potasio sérico y los parámetros electrocardiográficos, así como su interrelación en la alpaca.

\section{Materiales y Métodos}

\section{Lugar de Ejecución y Animales}

El presente estudio se llevó a cabo en comunidades alpaqueras del distrito de Maranganí, provincia de Canchis, departamento de Cusco, ubicadas por encima de los $3800 \mathrm{msnm}$. Los registros de los electrocardiogramas (ECG) en las alpacas se hicieron en las comunidades y las muestras de sangre se analizaron en la Facultad de Medicina Veterinaria (FMV), Universidad Nacional Mayor de San Marcos (UNMSM), Lima.

Se utilizaron 30 crías de alpacas (17 machos y 13 hembras), aparentemente sanas, entre 1 a 60 días de edad y criadas bajo las mismas condiciones de manejo y alimentación. La edad se determinó a través de los registros existentes en cada localidad. 


\section{Electrocardiografía (ECG)}

Los animales fueron sujetados, sin sedación, en decúbito lateral derecho. Se colocaron cuatro conectores, uno en cada extremidad. En los miembros anteriores, los conectores fueron colocados por encima del olecranon, a nivel del tercio distal del húmero, y en los miembros posteriores se colocaron por encima de la articulación femurotibiorotuliana. En cada caso, se aplicó alcohol para mejorar la conducción eléctrica.

Se tomó en cuenta la evaluación de derivadas unipolares (AVR, AVL y AVF) y derivadas bipolares (I, II y III), con énfasis sobre la derivada II, a velocidades de $25 \mathrm{~mm} / \mathrm{s}$ y $50 \mathrm{~mm} / \mathrm{s}$ y con milivoltaje $10 \mathrm{mv}$, a fin de abarcar un mayor mapeo o espacio del área cardiaca.

\section{Potasio Sanguíneo}

Se tomaron muestras de sangre $(2 \mathrm{ml})$ de la vena yugular en tubos sin anticoagulante. El suero fue separado y conservado en nitrógeno líquido y remitido a la FMVUNMSM, Lima. Los niveles de potasio se determinaron a través de cinética enzimática por luz ultravioleta con un kit comercial (Far Diagnostics, Italia), siguiendo el protocolo del fabricante. La lectura se hizo por medio de un espectofotómetro Spectro UV-VIs 2028 (Labomed Inc., California, EEUU).

Los valores se expresan como promedios y desviaciones estándar. Las posibles diferencias en los valores de potasio en sangre debidas al sexo se determinaron mediante la prueba de T-student con un nivel de confianza del $95 \%$.

\section{Resultados y Discusión}

El nivel de potasio en suero en las crías de alpaca fue de $5.91 \pm 1.48 \mathrm{mmol} / \mathrm{L}$, sin diferencias significativas entre sexos (machos: $6.18 \pm 1.45$; hembras: $5.56 \pm 1.49 \mathrm{mmol} / \mathrm{L}$ ). Así mismo, los valores oscilaron entre $2.1 \mathrm{y}$
$11.08 \mathrm{mmol} / \mathrm{L}$ para una hembra de 15 días y para un macho de 28 días de edad, respectivamente. Los valores de potasio sérico encontrados en el presente estudio fueron similares a los hallazgos en otros estudios, donde se reportan niveles de $5 \mathrm{mmol} / \mathrm{L}$ en terneros menores de tres meses (Maach et al., 1991), de $5.42 \mathrm{mmol} / \mathrm{L}$ en corderos (Ghanem y Abd El-Raof, 2005), y de $5.4 \pm 0.4 \mathrm{mmol} / \mathrm{L}$ en terneros (Bouda y Jagoš, 1984), aunque en este último caso, los valores decrecieron a partir del sexto mes. Por otro lado, Reece (1980) menciona un descenso de la concentración de potasio desde la semana $1(7.2 \mathrm{mmol} / \mathrm{L})$ a la semana $15(4.4 \mathrm{mmol} / \mathrm{L})$.

En los rumiantes, el potasio es absorbido en el rumen e intestino delgado y excretado por el riñón y en las heces. El 96 a 98\% del total de potasio se encuentra dentro de las células (Wörth, 1999) y altas concentraciones en el organismo pueden ser tóxicos. En terneros, el ingreso de calostro influye sobre el incremento de las concentraciones de potasio sérico, principalmente debido por su alta concentración en el calostro (Steinhardt et al., 1993).

En el Cuadro 1 se muestran los valores promedios de los valores electrocardiográficos. No se observó mayor variabilidad entre edades o sexo. La excepción fueron dos machos que presentaron bloqueo de grado II, pero sin sintomatología evidente de problemas cardíacos. Estos resultados presentan cierta similtud a los resultados de Mendes et al. (2001) y de Ghita et al. (2008), ambos en terneros, donde estos últimos consideran el registro del ECG de gran utilidad para la detección de enfermedades cardíacas en terneros (distrofia miocárdica, ampliaciones del corazón, derrame del pericardio).

Se pudo observar, además, que crías con la onda $\mathrm{T}$ aumentada presentaron valores de potasio sérico por encima de $5.5 \mathrm{mmol} /$ L; sin embargo, esta asociación no estuvo acompañada de alteraciones cardíacas. Diversos estudios mencionan que valores superiores a $5 \mathrm{mmol} / \mathrm{L}$ están asociados a un in- 
Cuadro 1. Valores electrocardiográficos en crías de alpacas entre 1 y 60 días de edad

\begin{tabular}{lll}
\hline Paráme tro ECG & \multicolumn{2}{c}{ Promedio \pm de } \\
\hline Duración onda P (s) & 0.040 & 0.0 \\
Amplitud onda P (mV) & 0.173 & 0.058 \\
Duración PQ (s) & 0.101 & 0.020 \\
Duración QRS (s) & 0.038 & 0.006 \\
Complejo QRS (mV) & 0.557 & 0.412 \\
Duración QT (s) & 0.237 & 0.040 \\
Amplitud onda T (mV) & 0.413 & 0.266 \\
\hline
\end{tabular}

cremento en la altura y ancho de la onda $\mathrm{T}$ en humanos (Brady, 2010; Mattu y Brady, 2008; Cameron et al., 2009; Hampton, 2008). La hiperkalemia (hiperpotasemia) se define como leve, moderada y severa cuando los valores de potasio sérico son entre 5.5 y 5.9 , entre 6.0 y 6.9, y mayores de $7.0 \mathrm{mmol} / \mathrm{L}$ (Wrenn et al., 1991; Martinez-Vea et al., 1999).

\section{Conclusiones}

- Los valores del potasio sérico y de los parámetros electrocardiográficos en alpacas menores de 60 días de edad muestran similitud con los valores de otras especies.

- El sexo no afectó los valores de potasio sérico en crías de alpacas.

\section{Literatura Citada}

1. Bouda J, Jagoš P. 1984. Biochemical and hematological reference values in calves and their significance for health control. Acta Vet Brno 53: 137-142.

2. Brady W. 2010. Hyperkalemia and the electrocardiogram. Emergencias 22:152.
3. Cameron P, Jelinek G, Kelly AM, Murray L, Brown AF. 2009. Textbook of adult emergency medicine. $3^{\text {rd }}$ ed. New York: Elsevier. 1028 p.

4. Ghanem M, Abd El-Raof Y. 2005. Clinical and haemato-biochemical studies on lamb coccidiosis and changes following amprolium and sulphadimthoxine therapy. Benha Vet Med J 16: 286-300.

5. Ghita M, Cotor G, Brãsla ${ }^{o}$ u C. 2008. The values of ECG parameters in new born calves. Bull UASVM Vet Med 65(1): 473.

6. Hampton JR. 2008. The ECG in practice. $5^{\text {th }}$ ed. Churchill Livingstone: Elsevier. 360 p.

7. Kittleson M, Kienle R. 1998. Small animal cardiovascular medicine. USA: Mosby. 83 p.

8. Maach L, Gründer HD, Faio A. 1991. Hämozytologische und hämobiochemische Untersuchungen bei schwarzbunten, klinisch gesunden Aufzuchtkälbern in Marokko. Deut Tierarztl Woch 98(3): 77116.

9. Martínez R, Urquieta B, Rojas J, Sumar, J. 1988. Estudio electrocardiográfico comparativo en camélidos sudamericanos en la Región Altoandina y a nivel del mar. Avances Med Vet 3(2): 9297.

10. Martinez-Vea A, Bardaji A, Garcia C. 1999. Severe hyperkalemia with minimal electrocardiographic manifestations: A report of seven cases. J Electrocardiol 32: 45-49.

11. Mattu A, Brady W. 2008. ECGs for the emergency physician 2. USA: BMJ Books. 224 p.

12. Mendes L, Camacho A, Alves A, Borges A, Souza R, Ferreira W. 2001. Standard electrocardiographic values in Holstein calves. Arq Bras Med Vet Zootec 53: 641- 644.

13. Nelson R, Couto G 1995. Pilares de medicina interna en animales pequeños. Buenos Aires: Interamericana. 977 p.

14. Reece WO. 1980. Acid-base balance and selected hematologic, electrolyte, and blood chemical variables in calves: milk- 
fed vs conventionally fed. Am J Vet Res 41: 109-113.

15. Seifter JL. 2012. Potassium disorders. In: Goldman L, Ausiello D (eds). Goldman's Cecil Medicine. 24 $4^{\text {th }}$ ed. Philadelphia: Elsevier. 734-741.

16. Steinhardt M, Gollnast I, Langanke M, Bünger U, Kutschke J. 1993. Klinisch-chemische Blutwerte bei neugeborenen Kälbern 1. Einflüsse eini- ger innerer und äußerer Bedingungen. Tierärztl Prax 21: 295-301.

17. Wörth W. 1999. Elektrolyte und Säure Basen - Haushalt. In: Kraft W, Dürr UM (eds). Klinische Labordiagnostik in der Tiermedizin 5. Stuttgart, Germany: Schattauer. p 159-168.

18. Wrenn KD, Slovis CM, Slovis BS. 1991. The ability of physicians to predict hyperkalemia from the ECG. Ann Emerg Med 20: 1229-1232. 